ADP-01-27/T461

\title{
Distortions in the negative energy Dirac sea: violation of the Gottfried sum rule and $\Delta \bar{u}$ in proton
}

\author{
K. Tsushima ${ }^{1 a}$, A.W. Thomas ${ }^{2}$ \\ Special Research Centre for the Subatomic Structure of Matter \\ and Department of Physics and Mathematical Physics, \\ Adelaide University, Adelaide SA 5005, Australia \\ E-mail: ${ }^{1}$ ktsushim@physics.adelaide.edu.au, ${ }^{2}$ athomas@physics.adelaide.edu.au \\ G.V. Dunne \\ Department of Physics, University of Connecticut, \\ Storrs, CT 06269-3046, USA \\ E-mail: dunne@phys.uconn.edu
}

\begin{abstract}
We report on recent work concerning the effect which the change in vacuum structure (negative energy Dirac sea), in the presence of a confining scalar field, has on the nucleon structure functions and parton distributions. Using the Dirac equation in $1+1$ dimensions, we show that distortions in the Dirac sea are responsible for part of the violation of the Gottfried sum rule - i.e., part of the flavor asymmetry in the proton sea. Our basic argument is that, even if isospin is an exact symmetry, the presence of a confining potential changes the vacuum structure, and inevitably leads to a violation of $\mathrm{SU}(2)$ flavour symmetry in a hadron with a different number of valence $u$ and $d$ quarks. The same mechanism also leads to a prediction for $\Delta \bar{u}$ and $\Delta \bar{d}$.
\end{abstract}

\section{Introduction}

The study of the violation of the Gottfried sum rule 1 has attracted considerable interest_since its experimental confirmation by the New Muon Collaboration $(\mathrm{NMC})$ 2. At present, there are several possible explanations for it, including mesonic (mainly pionic 3 ) contributions and the Pauli exclusion principle at the quark levell - for recent reviews see Refs.

Here we report our recent work 9 , on this problem, aimed at putting the phenomenon into a more general context. Our approach is based on a totally nonperturbative effect, namely the change in the vacuum structure (including distortion of the negative energy Dirac sea) in the presence of a confining potential. Such changes in vacuum structure have been studied jn oreat detail in other contexts (including the phenomenon of fractional charge $20,11,12$ ), since the initial work of Jackiw and Rebbi10.

${ }^{a}$ Presented at the Joint Workshop of the Special Research Center for the Subatomic Structure of Matter (CSSM) and the National Institute for Theoretical Physics on Lepton Scattering, Hadrons and QCD, March 26 - April 6, 2001, Adelaide, Australia. 
In the case of the nucleon structure functions, self-consistent studies of the vacuum structure have been made within a chiral quark soliton model $13,44.15 .16$. However, it is not easy to see how the change in vacuum structure is directly reflected in the calculated results.

In this report we will explicitly show that the existence of a (quark confining) potential distorts the negative energy Dirac sea and inevitably leads to the violation of the Gottfried sum rule. We emphasize that our argument holds even if charge symmetry and flavor SU(2) symmetry are assumed to be valid. We also predict $\Delta \bar{u}$ in proton based on the same mechanism.

\section{The Dirac equation in $1+1$ dimensions}

Consider massless $(m=0)$ fermions in $1+1$ dimensions, in the presence of a static scalar potential $V(z)$. The fermion spectrum is determined from the Dirac Hamiltonian,

$$
\begin{aligned}
H \varphi(z) & =\left[-i \alpha \frac{d}{d z}+\beta V(z)\right] \varphi(z), \\
& =\left(\begin{array}{cc}
0 & -\frac{d}{d z}+V(z) \\
\frac{d}{d z}+V(z) & 0
\end{array}\right) \varphi(z)=E \varphi(z),
\end{aligned}
$$

where we have chosen the Dirac matrix representation with $\alpha=\sigma_{2}$ and $\beta=\sigma_{1}$. We choose $V(z)$ to be an odd function, with a kink-like profile such that $V(\infty)=V_{0}=-V(-\infty)$. Then the spectrum is necessarily symmetric about $E=0$, and there are continuum thresholds at $E= \pm V_{0}$. There is a bound state at $E=0$, but it is irrelevant for our discussion. Instead, we consider bound states in the gap $|E|(\neq 0)<\left|V_{0}\right|$, which occur in pairs symmetrically placed about $E=0$.

We choose the profile for the scalar potential: $V(z)=V_{0} \tanh z$, where $V_{0}>0$. Then the $E \neq 0$ bound state wave functions with energy $E_{n}^{( \pm)}=$ $\pm \sqrt{n\left(2 V_{0}-n\right)}$, are given by

$$
\chi_{b, n}^{( \pm)}(z)=N_{n}\left(\begin{array}{c}
\sqrt{\frac{n}{2 V_{0}-n}} P_{V_{0}}^{V_{0}-n}(\tanh z) \\
\pm P_{V_{0}-1}^{V_{0}-n}(\tanh z)
\end{array}\right),
$$

for $n=1,2, \ldots\left[V_{0}\right]$, where $\left[V_{0}\right]$ means the greatest integer less than $V_{0}$. In Eq. (2), $P_{\nu}^{\mu}(z)$ is the associated Legendre function. If $V_{0}$ is an integer then there are real (but non-normalizable) threshold states obtained by setting $n=V_{0}$ in Eq. (2). The continuum state wave functions are given by

$$
\varphi_{p}^{( \pm)}(z)=N_{p}\left(\begin{array}{c}
\sqrt{\frac{V_{0}-i p}{V_{0}+i p}} P_{V_{0}}^{i p}(\tanh z) \\
\pm P_{V_{0}-1}^{i p}(\tanh z)
\end{array}\right),
$$


with energy $E_{p}^{( \pm)}= \pm \sqrt{V_{0}^{2}+p^{2}}$. For simplicity, we choose $V_{0}=\frac{3}{2}$, where there is a zero energy state, and two other bound states with energies $E_{1}^{( \pm)}= \pm \sqrt{2}$ :

$$
\chi_{b, 1}^{( \pm)}(z)=\left(\begin{array}{c}
\sqrt{2} \tanh z \operatorname{sech}^{1 / 2} z \\
\pm \operatorname{sech}^{1 / 2} z
\end{array}\right)
$$

These solutions are to be compared to the free Dirac equation solutions of positive and negative energies $\left(E_{p}= \pm|p|\right)$ :

$$
u_{p}(z)=\frac{1}{\sqrt{2}}\left(\begin{array}{c}
-i p /|p| \\
1
\end{array}\right) e^{i p z}, \quad v_{p}(z)=\frac{1}{\sqrt{2}}\left(\begin{array}{c}
i p /|p| \\
-1
\end{array}\right) e^{-i p z} .
$$

Then, the field operator can be expanded in terms of either $\left\{u_{p}, v_{p},-\infty<\right.$ $p<\infty\}$ or $\left\{\chi_{b} \varphi_{p}^{(+)}, \varphi_{p}^{(-)},-\infty<p<\infty\right\}$, each of which is a complete, orthonormal set 12 :

$$
\begin{aligned}
\psi(z) & =\int_{-\infty}^{\infty} \frac{d p}{2 \pi}\left[b_{p} u_{p}(z)+d_{p}^{\dagger} v_{p}(z)\right] \\
& =e_{n} \chi_{b, n}+\int_{-\infty}^{\infty} \frac{d p}{2 \pi}\left[a_{p} \varphi_{p}^{(+)}(z)+c_{p}^{\dagger} \varphi_{p}^{(-)}(z)\right] .
\end{aligned}
$$

The creation and annihilation operators for the set with $V_{0} \neq 0$ satisfy,

$$
\left\{e_{n}^{\dagger}, e_{m}\right\}=\delta_{n m},\left\{a_{p}^{\dagger}, a_{q}\right\}=2 \pi \delta(p-q),\left\{c_{p}^{\dagger}, c_{q}\right\}=2 \pi \delta(p-q),
$$

and all other anticomutators vanish. The operator sets, e.g., $\left\{e_{n}, a_{p}, c_{p}^{\dagger}\right\}$ and $\left\{b_{p}, d_{p}^{\dagger}\right\}$ can be related using the orthogonality (Bogoliubov transformation):

$$
\begin{aligned}
e_{n} & =\left\langle\chi_{b, n} \mid u_{p}\right\rangle b_{p}+\left\langle\chi_{b, n} \mid v_{p}\right\rangle d_{p}^{\dagger}, \\
a_{k} & =\left\langle\varphi_{k}^{(+)} \mid u_{p}\right\rangle b_{p}+\left\langle\varphi_{k}^{(+)} \mid v_{p}\right\rangle d_{p}^{\dagger}, \\
c_{k}^{\dagger} & =\left\langle\varphi_{k}^{(-)} \mid u_{p}\right\rangle b_{p}+\left\langle\varphi_{k}^{(-)} \mid v_{p}\right\rangle d_{p}^{\dagger},
\end{aligned}
$$

where the bra-ket notation implies, $\int_{-\infty}^{\infty} \frac{d p}{2 \pi}$.

Let us focus on the bound state, $\left|\chi_{b, 1}\right\rangle$, where the wave function is normalized to unity and its expression is given by Eq. (1). Using the relation of Eq. (9) and the property of orthonormality, the normalization of the bound state can be written by:

$$
1=\left\langle\chi_{b, 1} \mid \chi_{b, 1}\right\rangle=\int_{-\infty}^{\infty} \frac{d p}{2 \pi}\left(\left|\left\langle\chi_{b, 1} \mid u_{p}\right\rangle\right|^{2}+\left|\left\langle\chi_{b, 1} \mid v_{p}\right\rangle\right|^{2}\right) .
$$


In Eq. (12), the term $\left|\left\langle\chi_{b, 1} \mid v_{p}\right\rangle\right|^{2}\left(\left|\left\langle\chi_{b, 1} \mid u_{p}\right\rangle\right|^{2}\right)$, can be interpreted as the probability density distributions with which the bound state $\left|\chi_{b, 1}\right\rangle$ would be filled by the negative (positive) energy free states, if the potential were turned off suddenly (but not adiabatically).

We show in Fig. 1] the quantity $\left|\left\langle\chi_{b, 1} \mid u_{p}\right\rangle\right|^{2}+\left|\left\langle\chi_{b, 1} \mid v_{p}\right\rangle\right|^{2}$, (square of the wave function overlaps) as a function of energy for the free plane wave solutions, $E_{p}$ (free). Surprisingly, one can immediately notice that the positive

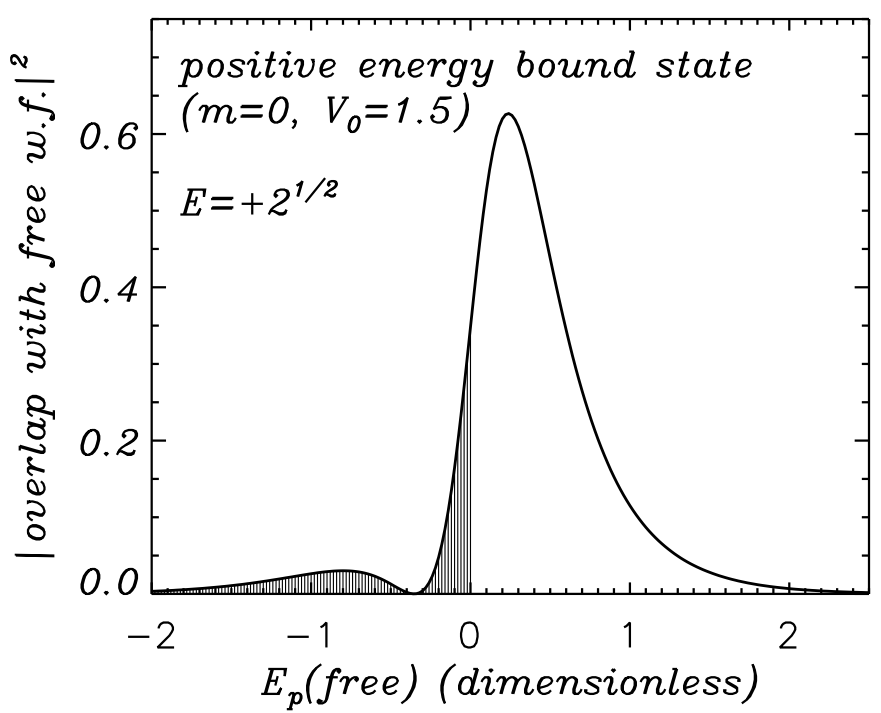

Figure 1: Bound state number density distributions as a function of free plane wave energy, $E_{p}($ free $)$, when projected onto free plane wave solutions.

energy bound state wave function, $\chi_{b, 1}$, has non-zero overlaps with the negative energy free plane wave solutions (the shaded area in Fig. 1). If we integrate over $E_{p}($ free $)$, it gives unity by construction, or probability conservation, as it should be. We should also mention that the negative (positive) energy continuum state wave functions of Eq. (3) have also non-zero overlaps with the positive (negative) energy free plane wave solutions.

The conclusion of this section is that the positive energy bound state, appearing in the Fock space built on the presence of the potential, has nonzero overlaps with the negative energy free plane wave solutions. It is this point that we want to make a connection with the deep-inelastic scattering (DIS) and the parton model. 


\section{Connection with the Parton Model}

In this section we consider the interpretation of the results obtained in the previous section in connection with the parton model. It may be useful to recall the assumptions which leads to the standard parton model according to Ref.17: (1) The current couples to quarks. Then the contributions to the forward Compton amplitude can be classified by the flow and interactions of quark lines. (2) At large value of $Q^{2}$ the currents, but not the states, may be treated as in free field theory. Thus, final state interactions and vertex corrections are ignored.

Let us now identify the positive energy bound state wave function of Eq. (1) as that of the valence quark in a simple, model proton. We recall that in Fig. 1 the quark number density distribution is projected onto free plane wave solutions of energy $E_{p}$ (free). As mentioned above, these free plane wave solutions are now to be identified with the partons, which appear in free field theory. As expected, the valence quark wave functions in the proton have non-zero overlap with the negative energy free plane waves, and this means that the distributions are non-zero in the region where Bjorken $x$ is negative. As a result the integral of the valence distribution over the physical region $x>0$ will not be one. The resolution of this problem lies in the fact that the Dirac sea in the presence of a scalar potential is also different from that of free space. In particular, the Dirac sea in the presence of the soliton in our 1D example (or the confining potential for the proton) consists of having all negative energy states occupied. If re-expressed in terms of free plane waves, however, one finds an equal number of positive energy free states occupied and negative energy states (holes) empty. That is, when the occupied negative energy states are expressed in terms of free states one finds an intrinsic sea of $q \bar{q}$ pairs. In the absence of valence quarks this sea carries no net flavour (or in $3 \mathrm{D}$, spin), relative to the free space vacuum.

However, once we add a single valence quark (say a $u$ quark), the negative energy tail will fill some of the holes in the (free space) Dirac sea. An equal number of occupied positive energy states from the $u$ quark Dirac sea will then be interpreted as the missing valence normalization (because $u_{v}$ is defined to be $u-\bar{u}$ ). The net result is that we have one valence $u$ quark, together with a reduction in the number of $u \bar{u}$ pairs in the sea of the proton. That is, one will have $\bar{d}>\bar{u}$ by an amount equal to the area under the valence curve to the left of $E_{p}=0$ in Fig. 1. If instead we had one valence $u$ and one valence $d$ the intrinsic sea would be flavour symmetric again, while for two valence $u$ 's and one valence $d$, as in the proton, we have $\bar{d}>\bar{u}$ by the same amount as for the single valence $u$ (the area under the valence curve to the left of $E_{p}=0$ in Fig. 


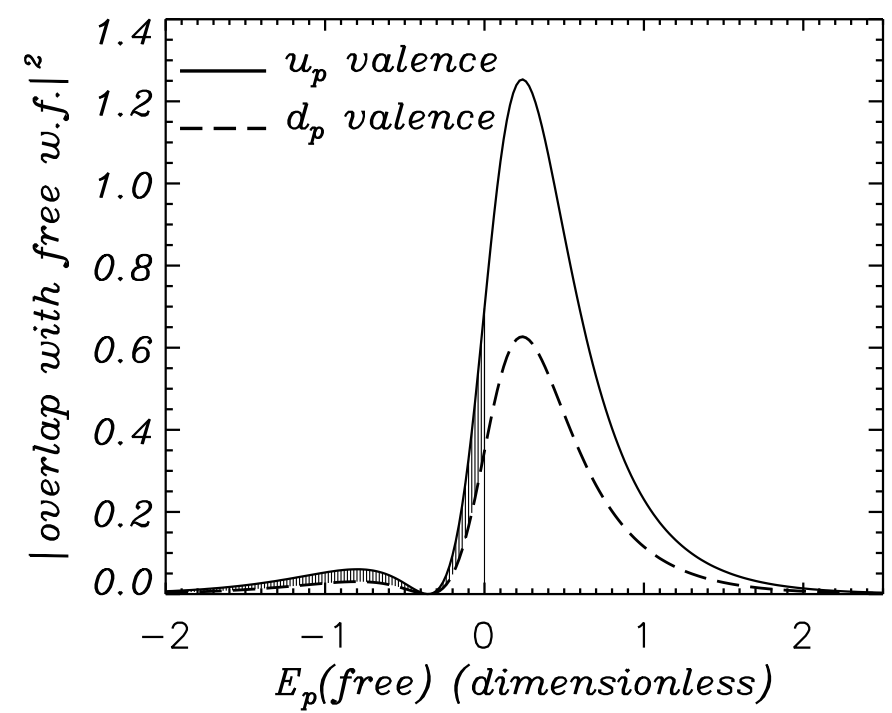

Figure 2: $u$ and $d$ valence quark number density distributions in the "proton" as a function of free energy, $E_{p}($ free $)$, projected onto free plane waves.

1).

The situation with respect to the Gottfried sum rule is therefore illustrated in Fig. 2. Because there are more valence $u$ 's, the $u$ sea is depleted more than the sea of $d$ 's. Mathematically, the expression for the Gottfried sum rule is then:

$$
\begin{aligned}
S_{G} & =\int_{0}^{1} \frac{d x}{x}\left[F_{2}^{\mu p}(x)-F_{2}^{\mu n}(x)\right], \\
& =\frac{1}{3} \int_{0}^{1} d x\left[u_{v}(x)-d_{v}(x)\right]-\frac{2}{3} \int_{0}^{1} d x[\bar{d}(x)-\bar{u}(x)], \\
& =\frac{1}{3}-\frac{2}{3} \int_{-\infty}^{\infty} \frac{d p}{2 \pi}\left|\left\langle v_{p} \mid \chi_{b, 1}\right\rangle\right|^{2} .
\end{aligned}
$$

(Note that we have assumed charge symmetry to set $d^{n}=u^{p} \equiv u$, etc.)

\section{Conclusion}

By considering the simple 1D problem outlined here, we aimed to make the physics origins of one source of flavour asymmetry in the proton explicit and 
easy to understand. While one cannot access spin in the 1D case, the simplicity of the argument for flavour makes the corresponding argument for $\Delta \bar{u}$ (and $\Delta \bar{d})$ clear. Since in a spin up proton described by an $\mathrm{SU}(6)$ wave function the valence $u$ quark is predominantly spin up, one will be missing spin up $u$ quarks in the sea of the proton. Thus $\Delta \bar{u}$ will be positive and of a similar magnitude to that of $\bar{d}-\bar{u}$ from the same source 18 . Since the $d$ quarks tend to have spin down in the proton, one also expects $\Delta \bar{d}$ to be negative and considerably smaller in magnitude than $\Delta \bar{u}$. Since any contribution to the observed flavour asymmetry from the nucleon's pion cloud is unpolarized 3.19 , the experimental determination of $\Delta \bar{u}$ and $\Delta \bar{d}$ would be extremely valuable in reaching a quantitative understanding of the roles of these two very different physical mechanisms in generating the non-perturbative structure of the proton sea.

\section{Acknowledgments}

We would like to thank W. Melnitchouk and A.W. Schreiber for many helpful discussions. K.T. would like to acknowledge the hospitality at KFA Jülich, where this report was completed. This work was supported by the Australian Research Council and Adelaide University. GD is supported by the US DOE, and thanks the CSSM at Adelaide for hospitality during a visit in which this work was begun. 


\section{References}

1. K. Gottfried, Phys. Rev. Lett. 18, 1174 (1967).

2. P. Amaudruz et al., Phys. Rev. Lett. 66, 2712 (1991); M. Arneodo et al., Phys. Rev. D50, R1 (1994).

3. A. W. Thomas, Phys. Lett. B 126, 97 (1983).

4. A. I. Signal and A. W. Thomas, Phys. Rev. D 40, 2832 (1989); A.W. Schreiber, PhD thesis, University of Adelaide, (1990); A. W. Schreiber, A. I. Signal and A. W. Thomas, Phys. Rev. D 44, 2653 (1991).

5. S. Kumano, Phys. Rep. 303, 183 (1998).

6. J. Speth and A.W. Thomas, Adv. Nucl. Phys. Vol. 24, 83 (1998).

7. J.T. Londergan, A.W. Thomas, Prog. Part. Nucl. Phys. 41, 49 (1998).

8. R. Vogt, Prog. Part. Nucl. Phys. 45, S105 (2000).

9. G.V. Dunne, A.W. Thomas, K. Tsushima, in preparation.

10. R. Jackiw and C. Rebbi, Phys. Rev. D13, 3398 (1976); see also, R. Jackiw, hep-th/9903255, Dirac Prize Lecture, Trieste, Italy - March 1999.

11. J. Goldstone and F. Wilczek, Phys. Rev. Lett. 47, 986 (1981); J. Goldstone and R. L. Jaffe, Phys. Rev. Lett. 51, 1518 (1983); For a review, see: A. Niemi and G. Semenoff, Phys. Rep. 135, 99 (1986).

12. R. MacKenzie and F. Wilczek, Phys. Rev. D30, 2194 (1984).

13. M. Wakamatsu, Phys. Rev. D44, R2631 (1991); Phys. Lett. B269, 394 (1991); Phys. Rev. D46, 3762 (1992).

14. D. Diakonov et al., Nucl. Phys. B480, 314 (1996).

15. P.V. Pobylitsa et al., Phys. Rev. D59, 034024 (1999); K. Goeke et al., Nucl. Phys. A680, 307 (2000); B. Dressler et al., Prog. Part. Nucl. Phys. 44, 293 (2000).

16. H. Weigel, E. Ruiz Arriola and L. Gamberg, Nucl. Phys. B560, 383 (1999).

17. R. L. Jaffe, in Relativistic Dynamics and Quark-Nuclear Physics, pp 537 - 618, M.B. Johnson and A. Picklesimer (Eds), (Wiley, New York, 1986).

18. F. Cao and A. I. Signal, hep-ph/0103113.

19. A. W. Thomas, W. Melnitchouk and F. M. Steffens, Phys. Rev. Lett. 85, 2892 (2000) hep-ph/0005043. 